Hilfsmittel in einem Kapitel vereinigt. Warum eigentlich diese recht praktische Einteilung aufgelassen wurde, ist nicht recht ersichtlich.

Die weitestgehende Umgestaltung erfuhr der Abschnitt über die Hauptbestandteile der Meßinstrumente. Es wird nicht nur der Theodolit und seine Bestandteile ausführlicher beschrieben, sondern es wird auch ein ganzes Kapitel über Entfernungsmesser und speziell über Doppelbildentfernungsmesser und neuere Hilfsmittel der Zentrierung eingeschaltet. Wesentlich verändert erscheint endlich auch das Kapitel über polygonometrische Messungen.

Dem Band ist ein kurzes Lebensbild des Verfassers W. J o r d a n vorausgestellt, welches aus der Feder seines Sohnes stammt und das recht traurige Schicksal beschreibt, welches die Schaffenskraft dieses Gelehrten frühzeitig lähmte. Er starb im Jahre 1899, erst 57 Jahre alt.

A. Prey.

G. H. A. Kröhnke, Taschenbuch zum Abstecken von Bögen bei Bahnen, Kanälen and Wegen. 17. Auflage, bearbeitet ron $R$. Seiffert, 133 Seiten $(12 \times 16 \mathrm{~cm})$ mit 21 Abbildungen. Verlag von B. G. Teubner, Leipzig und Berlin 1929.

Die Neuauflage der in der Fachwelt allgemein bekannten und beliebten Bogenabsteckungstabellen. von $\mathrm{Kröh} \mathbf{k} \theta$, welche im Jahre 1851 in erster Auflage erschienen sind und in den folgenden Auflagen eine den Bedürfnissen der Praxis Rechnung tragende Umgestaltung erfahren haben, weist gegenüber der letzten (16.) Auflage nur eine Erweiterung der erläuternden Einleitung auf, in welcher die für die verschiedenen Methoden der Kreisbogenabsteckung grundlegenden theoretischen Beziehungen zwischen den Elementen des Kreisbogens und den abzusteckenden Größen in übersichtlicher Weise zusammengestellt und auch die Absteckungsarboiten selbst und die dazu verwendeten Instrumente in einem für die Praxis hinreichenden Umfange behandelt werden. Die für die Absteckung der Kreisbogen erforderlichen Zahlenwerte sind in drei $Z$ ahlentafeln enthalten. Die erste dieser Zahlentafeln gibt die für die Absteckung der Hauptpunkte des Bogens (Bogenanfang, Bogenende und Bogenscheitel) erforderlichen Zahlenwerte für den Halbmesser 1000 und die Zentriwinkel von 0 bis 120 Grad von $10 \mathrm{zu} 10$ Minuten. Die Zahlentafel II enthält die Abszissen und Ordinaten äquidistanter Bogenpunkte brezogen auf die Tangente für alle vorkommenden Halbmesser von 20 bis 10.000 und die Zahlentafel III die Werte des Zentriwinkels im Gradmaße für die Bogenlängen 1 bis 9 und die in der Zahlentafel II vorkommenden Radien.

Die große Anzahl von Auflagen, welche die vorliegenden Tabellen erfahren haben, zeigen, daß sie den Anforderungen der Praxis vollkommen entsprechen und es ist daher nicht notwendig, dieses in der Praxis seit 80 Jahren eingebürgerte Hilfsbuch für die Bogenabsteckung noch besonders zu empfehlen.

Dokulil.

A. Morpurgo, Die wiederholte Einzelausgleichung. 26. Band von $T$ e u bn ers technischen Leitfäden. 45 Seiten $(13 \times 20 \mathrm{~cm})$ mit 3 Figuren im Text und 4 Tabellenbeilagen. Als Manuskript gedruckt. Verlag von B. G. Teubner, Leipzig und Berlin 1930. Preis kart. RM 3,40.

Der Verfasser bringt in dem vorliegenden Buche ein Näherungsverfahren zur vereinfachten Ausgleichung vermittelnder Beobachtungen mit vielen Unbekannten in Vorschlag, dessen Grundprinzip in einer wiederholten Einzelbestimmung der Unbekannten besteht. Die Unbekannten werden in einer für denselben Ausgleichungstall stets gleichbleibenden Reihenfolge derart wiederholt einzeln berechnet, daß bei der Berechnung einer Unbekannten alle übrigen erstmalig mit einem gewählten Näherungswerte, in der Folge aber mit dem jeweilig zuletzt ermittelten Werte als unveränderlich angenommen werden. Die Berechnung sämtlicher Unbekannten ist einzeln so lange zu wiederholen, bis die aus den Änderungen dreier aufeinanderfolgenden Werte einer Unbekannten berechneten Quotienten für sämtliche Unbekannten nur mehr solche Differenzen aufweisen, dio im Hinblicke auf den gegebenen $Z$ weck vernachlässigt werden können. Mit den so erhaltenen letzten Werten der Unbekannten wird eine nochmalige Einzelberechnung derselben vorgenommen und die dadurch erhaltenen Daten können dann als wahrscheinlichste Werte angenommen werden. 
Dieser in der Schrift allgemein behandelte und durch ausgeführte Beispiele über die Ausgleichung von Triangulierungs-, Nivellement- und trigonometrischen Höhennetzen erläuterte Vorgang hat gegenüber der strengen Ausgleichung den Vorteil, daßs die Rechenarbeit ungefähr proportional der Anzahl der Unbekannten ist, während sie bei der strengen Ausgleichung infolge der langwierigen Auflösung der Normalgleichungen ungefähr proportional dem Quadrate der Anzahl der Unbekannten angenommen werden kann. Die in den durchgeführten Beispielen nach fünf- bis sechsmaliger Wiederholung der Einzelberechnung erhaltenen Resultate zeigen eine fast vollständige Übereinstimmung mit den Engebnissen der strengen Rechning; insbesondere zeigt der Vergleich der nach beiden Rechnungsmethoden erhaltenen Fehlerquadratsummen, daß die im Wege des vorgeschlagenen Näherungsverfahrens abgeleiteten Werte der Unbekannten jenen der strengen Rechnung an Genauigkeit nicht nachstehen.

Das vorgeschlagene Rechnungsverfahren ist durch die wesentliche Vereinfachung der Rechnungsarbeit geeignet, die Anwendung der Methode der kleinsten Quadrate zur praktischen Durchfïhrung größerer Ausgleichungsprobleme zu fördern und dadurch der Verbreitung zweifelhafter empirischer Ausgleichungsverfahren, zu denen im Hinblicke auf die langwierige Arbeit bei der strengen Berechnung oft gegriffen wird, entgegenzutreten. Es kann das kleine, aber beachtenswerte Buch allen jenen, die sich mit der Praxis der Ausgleichungsrechnung befassen, bestens empfohlen werden.

Dokulil.

H. Oberth, Wege zur Raumschiffahrt. 3. Auflage von ,Die Rakete zu den Planetenräumen“". 442 Seiten. R. Oldenbourgh, München und Berlin 1929. Preis geh. RM 17,50, geb. RM 20,- .

Man könnte zunächst im $Z$ weifel sein, ob ein Buch wie das genannte überhaupt an dieser Stelle besprochen werden soll. Der Inhalt desselben besteht nämlich zum Teil aus phantasievollen. Betrachtungen über die Verhältnisse und Möglichkeiten bei einem Flug in den Weltenraum, aus Schilderungen der konstruktiven Details von verschiedenen mit flüssigem Brennstoff betriebenen Raketenmodellen, die der Verfasser erbauen möchte, und aus damit zusammenhängenden technisch-physikalischen Darlegungen. Es soll daher hier nur zu den theoretischen Berechnungen, die in dem Buche vorkommen, Stellung genommen werden.

Da ergeben sich sofort schwerwiegende Mängel. Statt für irgend ein Ausstoßungsgesetz der Masse für die Bewegung im luftleoren Raum unter Annahme der Schwerkraft die dynamischen Grundgleichungen anzusetzen, was das Gegebene wäre, wendet der Verfasser zunächst den Impulssatz bei Abwesenheit äußerer Kräfte an, führt dann ganz unsystematisch eine verzögernde Kraft ein und geht hierauf sofort zu ganz unübersichtlichen und unklaren Rechnungen über die Bewegung in der Luft über, die ja erst in zweiter Linie in Betracht käme. Deswegen übersieht er ganz, daß die Bewegung der Rakete wesentlich von der Art der Ausstoßung der Masse abhängt und kommt daher nicht zu richtigen Endresultaten. Diese sind leicht zu finden und zeigen, daß ein Erreichen einer Geschwindigkeit von $12 \mathrm{~km} / \mathrm{sek}$, die zum Verlassen der Erde notwendig wäre, Auspuffgeschwindigkeiten erforderte, die mit den heutigen Mitteln der Technik auf keine Weise erhalten werden können, wenn man ein halbwegs mögliches Verhältnis von Anfangs- und Endmasse haben will. Mit diesem prinzipiellen Fehler der nicht konsequenten Anwendung der dynamischen Grundgleichungen bei veränderlicher Masse hängt es auch zusammen, daß in den ,energetischen Betrachtungen" zum Teil Behauptungen aufgestellt werden, die unrichtig sind, wie die, daß der Satz von der Erhaltung des Sehwerpunktes sich aus dem von der Konstanz der Energie herleiten lasse. Der Verfasser ist sich selbst über die prinzipiellen Begriffe nicht ganz klar, wenn er auch zum Teil unrichtige Einwände in richtiger Weise zurückweist.

\section{K. Wolf.}

G. Ribaud, Température des Flammes. Rayonnement des Gaz incandescents et des Flammes. Conférences d'actualités scientifiques et industrielles $X$, 43 Seiten. Hermann \& Cie., Paris 1930, Preis geh. Frs. 5,-.

Zwei Vorträge im ,Conservatoire national des arts et métiers" über die Temperatur und Strahlung der Flammen. Der erste bringt die thermo- 